\title{
The Nematoda Filarioidea: CRITICAL ANALYSIS LINKING MOleCULAR AND TRADITIONAL APPROACHES
}

\author{
BAIN O.*, CASIRAGHI M.**, MARTIN C.* \& UNI S.***
}

\section{Summary:}

The molecular analysis of the Filarioidea and the endobacteria Wolbachia is no more limited to the agents of human diseases and the diversified sampling permits a synthesis with the morphological and biological results. The validity of the genera with "uncoherent host range", such as Mansonella, Litomosoides and Cercopithifilaria, is confirmed and, consequently, their evolution by host-switchings. Dirofilaria and Onchocerca, types of two subfamilies, appear more closely related than with other onchocercids. Waltonellinae from anurans and Oswaldofilariinae from reptiles have a basal position. These filariae, and some others also considered primitive, do not harbour Wolbachia. Evidence for transversal transmission of the bacteria and a second acquisition event is given with the supergroup $F$, identified in Mansonella, in one of the Cercopithifilaria species and in arthropods.

KEY WORDS : Filarioidea, Onchocerca, Dirofilaria, Dipetalonema, Wolbachia, phylogeny.

F ilarioids are parasitic nematodes extensively studied because several species are the agents of human diseases. The need to elucidate the epidemiological conditions of transmission and the requirement for relevant experimental models have stimulated a widespread zoological investigation. Our present concern is the systematics of the superfamily Filarioidea with emphasis on its generic composition, the relationships between genera, the validity of the supra-generic divisions, the roots and epoch of emergence of Filarioidea, related to the question of mono- or polyphyly. Two main investigative tools are currently available, the traditional morphological and biological, and the more recent molecular analyses. They are commonly used by independent specialized teams, with the risk of uncontrolled divergent interpretations for the same biological object. On the other hand, the combining of their respective results may foster more appropriate interpretations.

\footnotetext{
* Parasitologie Comparée et Modèles Expérimentaux, USM 307, Muséum National d'Histoire Naturelle, 75231 Paris Cedex 05, France.

*** Dipartimento di Biotecnologie e Bioscienze, Università degli Studi di Milano Bicocca, Piazza della Scienza 2, 20126 Milano, Italy.

*** Department of Medical Zoology, Osaka City University Medical School, Osaka 545-8585, Japan.

Correspondence: Odile Bain - E-mail: bain@mnhn.fr
}

The traditional tool includes the morphological characters of the adult and larval phenotypes, the biological data provided by vectors and experimental rodent models of filariases, as well as the host range and geographical distribution of worms. Since Wehr (1935), several classifications have been built on these data and the most recent (Anderson \& Bain, 1976), seemed to provide a correct synthesis; however new features, such as the discovery of genera or the better knowledge of larval development, have revealed several problems.

Nowadays, molecular analyses are available for a rather important sample of filariae, including also the study of the endosymbionts Wolbachia sensu lato (Casiraghi group and collaborators, work in progress). The genes studied are $5 \mathrm{~S}$ rDNA, 12S rDNA and coxI, less often 185 rDNA.

Our aim is to compare the results of both approaches with some examples chosen to distinctly highlight confirmation or contradiction of previous interpretations, and finally to delineate some unresolved problems.

\section{EXAMPLES OF CONGRUENCE BETWEEN MOLECULAR AND TRADITIONAL ANALYSES}

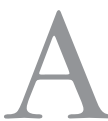
mong the filariae, there are several genera of which the host range is "zoologically incohe- Crent" (Chabaud, 1981, see Chabaud \& Bain, 1994). They are used to argue a model of evolution through host-switchings (captures), in opposition to a parallel evolution of the hosts and their parasites. However it may be objected that the genus is not welldelineated and, in fact, is a non-natural grouping of species. The molecular analyses should decipher such artefacts.

The congruence of the two methods of analyses appears quite good for the three genera studied Cercopithifilaria Eberhard, 1980, Litomosoides Chandler, 1931 and Mansonella Faust 1929, parasitic in mammals.

Cercopithifilaria is known with 28 species described from monkeys, ruminants, carnivores, lagomorphs, por- 
cupine and murid rodents, marsupials and monotremes. The small subunit of mitochondrial ribosome (12S rRNA) was analysed for nine species, from African porcupine hystricid, Japanese bear, Japanese bovids and cervids. The taxon, which had been created for a parasite of African cercopithecid monkeys, is actually a group of species having a large host-range and a worldwide distribution. As a matter of fact, the $12 \mathrm{~S}$ rDNA analysis confirms the value of the traditional characters used to define Cercopithifilaria (Fig. 1), the adult morphology and the infective larvae with no or minute buccal cavity. In addition adults have dermal or sub-cutaneous localizations, and skin-dwelling microfilariae. The vectors are the hard ticks Rhipicephalidae and it is hypothesized that they had the major role in the spread and diversification of Cercopithifilaria. In Australia, C. johnstoni Spratt \& Varughese, 1975, type host-Muridae, is found in marsupials and monotremes as well (communication by D. Spratt) and this might represent the first step of future speciations. Interestingly, Wolbachia is absent from species of the genus, except C. japonica (Uni, 1983) from bears, which harbours the form $\mathrm{F}$ of the endosymbiont (work in progress), a Wolbachia type shared with filariae of the genus Mansonella and with some arthropod hosts (Casiraghi et al., 2005).

32 species have been described in Litomosoides, from microchiropterans, marsupials, murids and a few other rodents. The $12 \mathrm{~S}$ rRNA was analysed for five species, three from bats and two from murids. The monophyly is also proved in this case. A particular morphological character of the cluster is the large segmented buccal capsule, about $20 \mu \mathrm{m}$ long in the adult worm and third stage larva (Fig. 1). The vectors are macronyssid acarians. Litomosoides is confined to the New World and has intensely diversified in the Neotropical region, probably by means of the mites. In the Old World, another genus has a large segmented buccal capsule and is parasitic in microchiropterans, and a common origin is supposed for the two genera. Indeed, a species parasitc in North American rodents Geomyioidea, Litomosa westi (Gardner \& Schmidt, 1986), which was initially placed in Litomosoides, would represent a link. This hypothesis is supported by the 12S rDNA analysis (Casiraghi et al., 2004; work in progress). Wolbachia is present in Litomosoides, with one exception (L. yutajensis Guerrero, Martin \& Bain, 2003) and belongs to the supergroup D; Wolbachia of Litomosa westi appears to belong to the same supergroup (work in progress). Mansonella Faust, 1929 contains 29 species, among which three are human parasites: one in South America, M. (M.) ozzardi (Manson, 1897), one in Africa, M. (Esslingeria) streptocerca (Macfie \& Corson, 1922), and one with an African origin subsequently introduced to South America since a few centuries, M. (E.) perstans (Manson, 1891). Two important diversifications are observed in anthropoid and platyrhinian monkeys. Other hosts are insectivores, including tupaids, carnivores and sciurid and caviomorph rodents (Eberhard \& Orihel, 1984). Recently, ruminant ungulates have joined this large host-range with species initially assigned to Cutifilaria Bain \& Schulz-Key, 1974, now a subgenus of Mansonella. The $5 \mathrm{~S} \mathrm{rDNA}$ and/or $12 \mathrm{~S}$ rDNA analyses (Xie et al., 1994; work in progress) have been performed with one species of Cutifilaria and three species from primates belonging to the subgenera Mansonella, Esslingeria Chabaud \& Bain, 1976 and Tetrapetalonema Faust, 1935, respectively. They cluster together and this correlates with distinctive morphological characters in adult worms: the oesophagus is thread-like, its blurred anterior end fused with the muscular body layer, and no buccal capsule is identifiable (Fig. 1). Wolbachia was not detected in M. (E.) perstans (Grobusch et al., 2003) but was detected in the other three species; it belongs to the supergroup $\mathrm{F}$ of the endosymbiont (work in progress).

\section{IS THE DIPETALOMEMA LINEAGE A REALITY?}

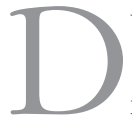
uring the past 30 years, Dipetalonema Diesing, 1861 has been split into several subgenera, many elevated to the generic rank, and the whole encompassed in the "Dipetalonema line". A common history from a Gondwanian ancestor during the late Second era was proposed for all of them, followed by divergences in the different regions separated by the continental drift (Chabaud \& Bain, 1976, in Chabaud \& Bain, 1994). In the "Dipetalonema line" males display a basic arrangement of the caudal sensory system (four precloacal pairs, pairs 5 and 6 post-cloa$\mathrm{cal}$, and a posterior group of pairs 7 to 10); the area rugosa, a cuticular anti-slit apparatus used during mating, is generally present. However the lineage is composed of plesiomorph and derived species (such as Acanthocheilonema and Cercopitbifilaria, respectively), and a clear definition was difficult. The present question is: do the molecular data support the Dipetalonema lineage?

Among its 17 genera and sub-genera, half of them have been submitted to molecular analyses: Acanthocheilonema Cobbold 1870, Dipetalonema, Cercopithifilaria, and Mansonella with four of its sub-genera, Mansonella, Esslingeria Chabaud \& Bain, 1976, Tetrapetalonema Faust, 1935, and Cutifilaria.

Acanthocheilonema and Dipetalonema sensu stricto are generally viewed as a cluster. The first genus contains parasites of carnivores, macroscelid insectivores and rodents, the second is currently restricted to parasites of South American platyrhinian monkeys. The studied species are $A$. reconditum (Grassi, 1889) from dogs, 


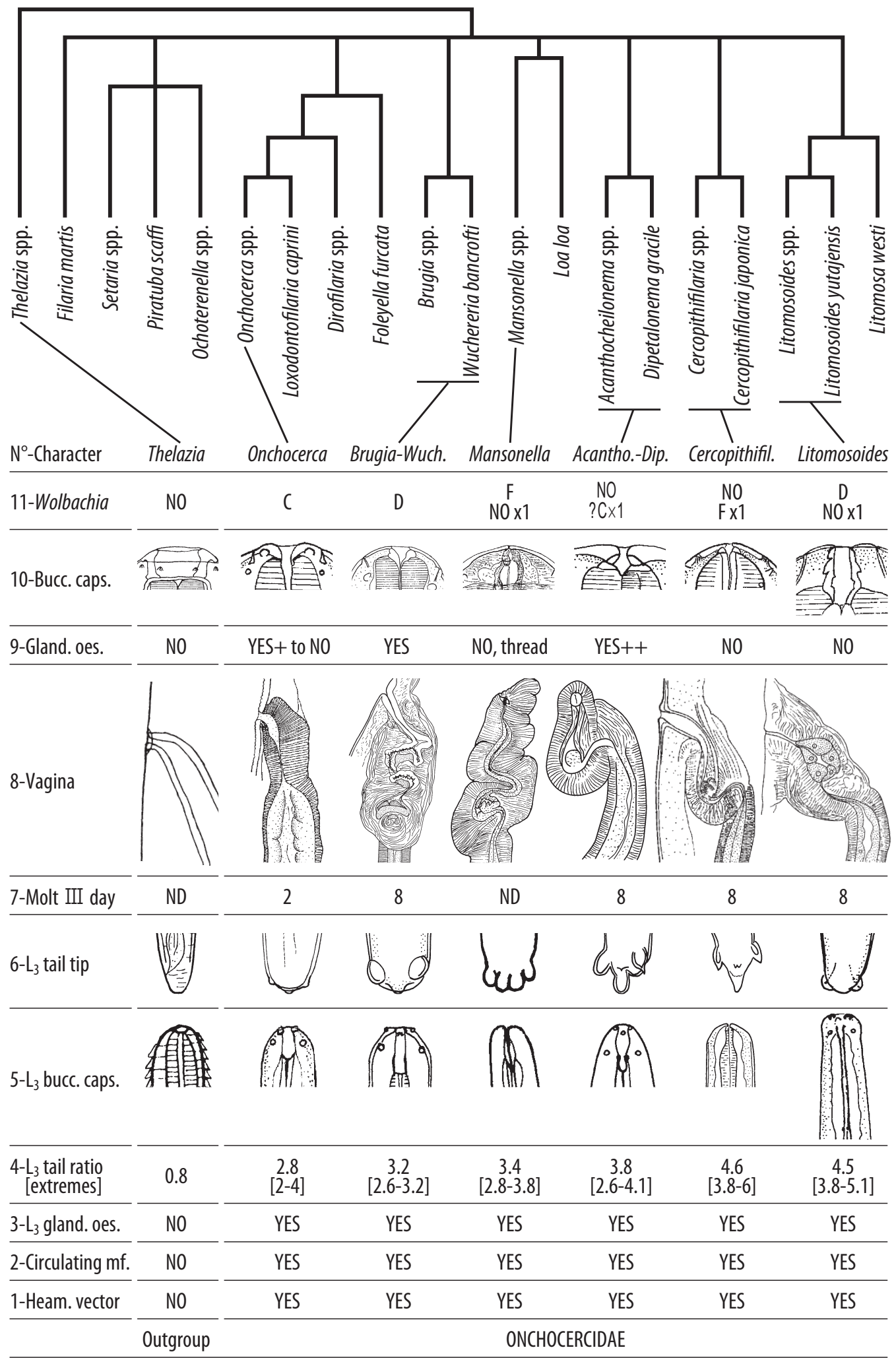

Fig. 1. - Filarioidea and the outgroup Thelazia: schematic illustration of molecular analysis and traditional data for some important genera. Distinctive characters selected are 1. Arthropod vector, Heamatophagus/not; 2. Biology of microfilariae, circulating/not; 3. L3 oesophagus, glandular part present/not; 4. L3, mean tail ratio length/width at anus [extremes]; 5. L3, buccal capsule, conspicuous/absent/very long; 6. L3, tail extremity; 7. Molt III, day 2 p. i./day 8 p. i.; 8. Vagina: no bend \& no chamber/bends/bends and conspicuous chamber; 9. Adult filaria, oesophagus, glandular part present (Yes to ++)/no; 10. Adult filaria, buccal capsule, 2 rings (or segments) $/ 1$ ring/absent/4 rings; 11. Wolbachia: absent (NOx1 means 1 species without Wolbachia) or a supergroup. The molecular tree presented is a "consensus" of the reconstruction generated in this and previous works. Larval characters mainly from Bain \& Chabaud (1986). 
A. viteae (Krepkogorskaya, 1933), used as a rodent model for filariasis, and D. gracile (Rudolphi, 1809). This cluster appears morphologically defined: the adult worms possess an oesophagus with a long thick glandular part, and the infective larvae have a buccal capsule 6$10 \mu \mathrm{m}$ long (Fig. 1). Cercopithifilaria is thus clearly different from the Acanthocheilonema-Dipetalonema cluster at the larval stage (no buccal capsule) and Mansonella at the adult stage (thread-like oesophagus). The well-developed caudal lappets of the infective larva of the four genera, which was one of their common characters, appears to be a convergence.

Filarioids from elephants, Loxodontofilaria Berghe \& Gillain, 1939, were not well known and provisionally placed in the Dipetalonema line (Bain, Baker, Chabaud, 1982, in Chabaud \& Bain, 1994). With the recent discovery of a species of Loxodontofilaria in a caprine (Uni et al., 2006), a molecular analysis was performed which lead to a different interpretation. It indicates close relationships with Onchocerca Diesing, 1841 (work in progress), although the vagina of Loxodontofilaria has distinct bend and chamber, contrary to Onchocerca. Loxodontofilaria also harbours Wolbachia of the group C, very similar to the endosymbionts harboured by members of the genus Onchocerca. Interestingly, Wolbachia is absent in the two Acanthocheilonema species studied (Casiraghi et al., 2004). According to the hypothesis proposed by Chabaud \& Bain (1994), these would be close to the roots of Gondwanian Acanthocheilonema-like filariae from which stemmed Dipetalonema s. s. in the Neotropical region, when platyrhinian monkeys arrived after their transoceanic migration (late Eocene). D. gracile, the single species studied in this derived branch, harbours a Wolbachia related to the $\mathrm{C}$ supergroup, even though its real assignation is still under discussion (see below and Casiraghi et al., 2004; 2005).

\section{NEW LIGHT ON THE ONCHOCERCINAE AND DIROFILARINAE}

W ithin the past 15 years, several molecular analyses of Onchocerca Diesing, 1841 and Dirofilaria Railliet \& Henry, 1910 were conducted, with ribosomal genes 5 S (Xie et al., 1994), coxI (Casiraghi et al., 2001), 12S rDNA (Casiraghi et al., 2004). All these molecular analyses placed the two genera close together. However each genus is presently the type of a subfamily (Anderson \& Bain, 1976). Dirofilariinae are distinguished from Onchocercinae by the shorter male tail, well-developed lateral alae and pedunculated caudal papillae.

A first disturbance to the classical systematics was provoked by Loa Stiles, 1905, Dirofilariinae: 5 S rDNA analysis placed Loa close to Mansonella, Onchocercinae
(Xie et al., 1994). Reconsideration of the morphological characters of Loa revealed that its position close to Dirofilaria was no longer sustainable: the infective stage of Loa is distinguished from that of Dirofilaria by possession of a long tail with well-developed caudal lappets, like Acanthocheilonema, Cercopithifilaria, and other onchocercids. Later, biological evidence strengthened the new interpretation. Larval development in Loa, Onchocerca, Dirofilaria, as well as in Brugia Buckley, 1958, Acanthocheilonema, Litomosoides and Monanema Anteson, 1968 were compared and demonstrated two strategies of worm establishment in the definitive host (Bain et al., 1997): the third moult occurs as soon as day 2 post-inoculation in Onchocerca and Dirofilaria but not until the end of the first week in the other genera, Loa included.

Molecular analysis has been carried out with two species of Dirofilaria, D. immitis (Leidy, 1856) and D. repens Railliet, 1911 from dogs, and many species of Onchocerca (Krueger et al., 2007; Sreter et al., 2007). However, only two studies encompassed several other genera of filarioids (Casiraghi et al., 2004; work in progress). The cluster Dirofilaria and Onchocerca is composed of two main branches, each corresponding to a genus. The cluster and its dichotomy concur with the morphological characters: the buccal capsule is reduced to an inconspicuous lamina in the adults, and is normally developed in the infective larvae ( 4 to $9 \mu \mathrm{m}$ long); these have a cylindrical tail with rounded extremity and tiny caudal lappets in both genera. However the tail of Dirofilaria larvae is short (ratio length/width less than 1) in contrast to that of Onchocerca, and the females of the 33 Onchocerca species (Uni et al., 2007) possess a simple vagina, without bend and chamber (Fig. 1) in contrast to Dirofilaria.

Dirofilaria harbours the group C of Wolbachia. Onchocerca harbours the same supergroup, when present. O. flexuosa, the single species without Wolbachia, displays a particular morphology and was assumed to be an "ancient" representative of the genus; this is congruent with the molecular datasets (Krueger et al., 2007). The other Onchocercinae listed above harbour the supergroups D (most of the cases) or F (in the case of Mansonella spp. and Cercopithifilaria japonica).

\section{POSITION OF THE SPECIES PARASITIC IN ANURANS AND REPTILES IN THE EVOLUTION OF FILARIOIDEA}

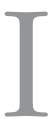
cosiellinae Anderson, 1958 and Waltonellinae, Bain \& Prod'Hon, 1974, with one and four genera respectively, are parasites of anurans. They are classically considered ancient, the first subfamily because the infective larva possesses cephalic spines, the second because 
of its Gondwanian host-range. The $12 \mathrm{~S}$ rDNA analysis performed on two Ochoterenella species confirms the basal position of the Waltonellinae (work in progress). Onchocercids parasitic in reptiles are dispersed among several genera and subfamilies. Their heterogeneity has been confirmed with the two species analyzed, an Oswaldofilariinae Chabaud \& Choquet, 1953 (Piratuba Freitas \& Lent, 1947), and a Dirofilariinae (Foleyella Seurat, 1917). The first has the most basal position and the second clusters with Dirofilaria and Onchocerca. The Oswaldofilariinae have several morphological characteristics: the infective larvae have longitudinal cuticular body crests; the vulva opens at midbody or posteriorly; however the female genital primordium migrates anteriorly during morphogenesis in the larval stage, as in other onchocercids. No Wolbachia has been identified in any of these filarioids from anurans and reptiles (Casiraghi et al., 2004; work in progress).

It is noted that Setaria Viborg, 1795 parasitic in ungulates is often placed close to Oswaldofilariinae and Waltonellinae near the base of the molecular phylogenetic trees. Setaria does not harbour Wolbachia, alike the outgroup genus Thelazia Bosc, 1819 (Casiraghi et al., 2004; work in progress).

\section{WOLBACHIA AND THE FILARIOIDEA}

S ince the identification of the bacteria Wolbachia in the tissues of some human filariae, a broader range of filarioid species has been studied resulting in a number of new features (work in progress). The assessment of Wolbachia omnipresent in infected species and its exclusive vertical transmission needs to be revised as well as the evolution of Wolbachia-filaria relationships.

In the human filariae O. volvulus, B. malayi and $\operatorname{dog}$ filaria D. immitis, the tissular distribution of Wolbachia includes the hypodermis of the lateral chords but this is not a general feature; in the animal filariae Loxodontofilaria caprini, M. (Cu.) perforata and O. dewittei japonica, Wolbachia is not detected in the hypodermis. Moreover, the prevalence of the bacteria in a given host species does not reach $100 \%$ in all cases; absence/ presence is observed in the three last species cited above and in O. skrjabini, although Wolbachia occurs in the lateral chords in this species and thus is expected to be positive regardless of which part of the worm is used for PCR. These data suggest different life styles of the wolbachiae and mutualistic relationships.

Acquisition of the mutualist through lateral transfer was demonstrated with a species of Cercopithifilaria, C. japonica, which harbours the supergroup $\mathrm{F}$ shared with Mansonella, and some insects (Lo et al., 2002; work in progress), whereas all other species of Cercopithifilaria have no Wolbachia.
A fact that recently has come to light is that the Onchocercidae which are considered primitive on the basis of traditional morphological and biological data have no Wolbachia. The list includes the Oswaldofilariinae and Waltonellinae, species of Foleyella (Dirofilariinae) parasitic in cold-blooded vertebrates, and the Setariinae. The case of $O$. flexuosa might be interpreted also as a primitive state in Onchocerca, or might be explained by a secondary loss of the endosymbiont. This highlights the fact that identification of a loss of Wolbachia may be problematic. However such regressive evolution seems well-established with Litomosoides: the endosymbiont is present in five of six species studied and L. yutajensis, which lacks the endosymbiont, has no morphological particularity.

The genus Wolbachia was divided into supergroups encompassing symbionts of arthropods (A, B and E) or nematodes ( $\mathrm{C}$ and $\mathrm{D})$. This picture changed when a further supergroup was described (F), which encompasses Wolbachia from both arthropods (in particular termites) and filarial nematodes. Additional molecular diversity in Wolbachia has been found in Australian spiders (supergroup G), other termites (supergroup H), fleas and potentially in the filarial nematode Dipetalonema gracile (Casiraghi et al., 2004).

Wolbachia is patchily distributed, leading to a complicated picture (Fig. 2). At the beginning of the evolutionary radiation of Wolbachia, around 100 mya (Bandi et al., 1998; Fenn et al., 2006) transfer of this endosymbiont from arthropods to nematodes (or vice versa) must have occurred. It is coherent with an ancestral absence of the endosymbiont in deep branches of Filarioidea, followed by at least one acquisition in the lineage leading to the derived species Onchocerca and Dirofilaria, the same or another acquisition leading to many other Onchocercinae. The strict relationship of Wolbachia from arthropods and nematodes in the F supergroup shows that a similar transfer might also have occurred more recently and independently from the ancestral host switch (work in press).

\section{UNRESOLVED QUESTIONS}

T The present critical analysis stresses the general congruence between traditional and molecular approaches at the generic rank in the Filarioidea. This validates the morphological and biological characters selected for diagnosis (Fig. 1). The major unresolved questions are the delineation of the Filarioidea, their origin and evolution.

Larval characters are reliable for the suprageneric relationships since ontogeny reflects phylogeny in Spirurida. The character "glandular part of eosophagus present in the infective stage" encompasses the Filarioidea 


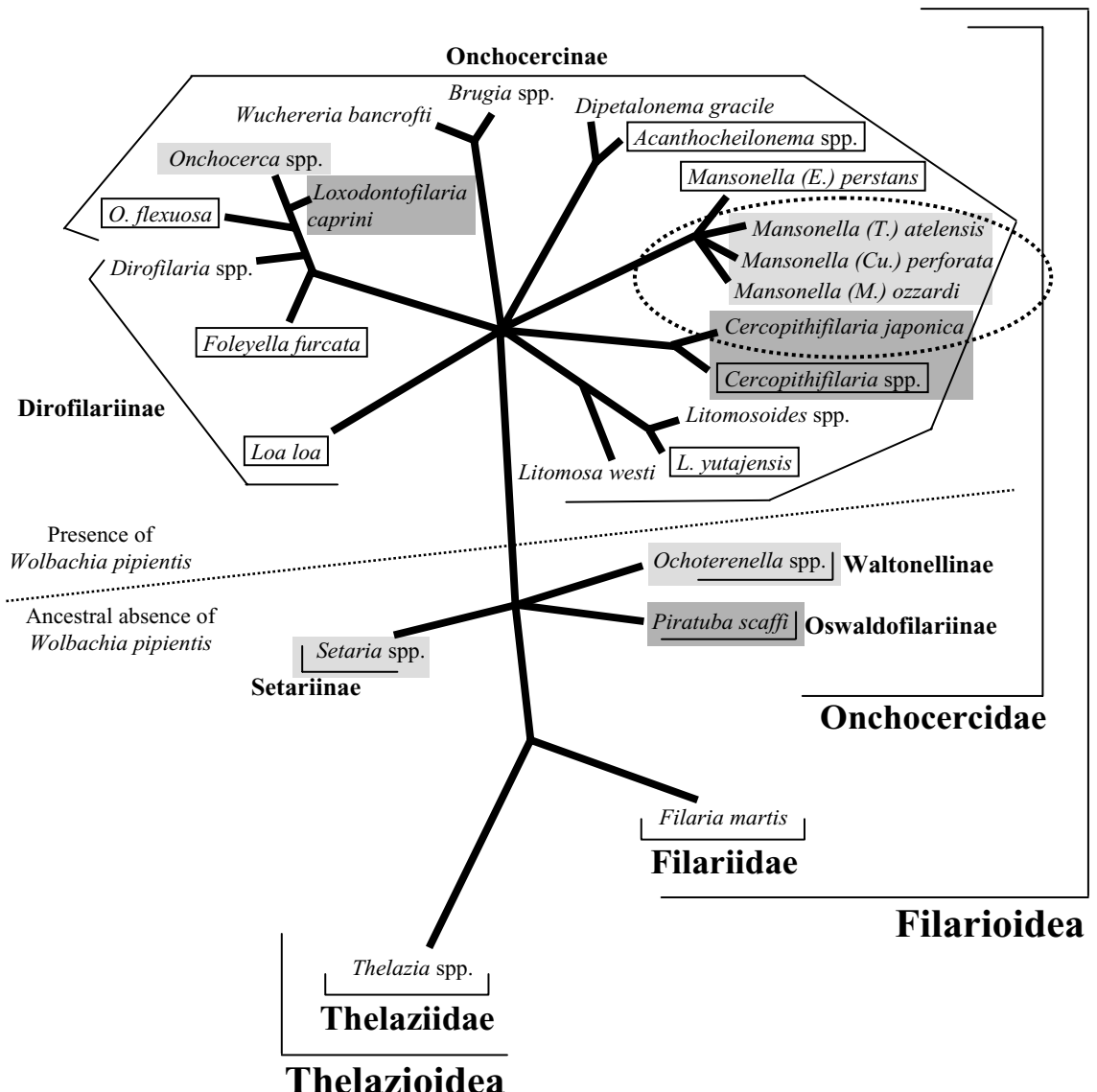

Fig. 2. - Hypothetical evolution of Wolbachia infection mapped on the phylogenetic tree of filariae and related nematodes. The present data suggest that Wolbachia was ancestrally absent from the lineages leading to Thelaziidae, Filariidae, Setariinae, Oswaldofilariinae and Waltonellinae, these two last parasitic in cold blooded vertebrates. Wolbachia was acquired on the lineage leading to the Onchocercinae + Dirofilariinae, diverging into supergroups C and D. C. japonica and Mansonella spp. (outlined in dashed ellipse) share a common supergroup F Wolbachia, due to a secondary acquisition. Losses along some lineages presented here (outlined in boxes) are more controversial and, except for Litomosoides yutajensis and Loa loa, could also be explained by a remote lineage birth, as for Foleyella from saurians, Acanthocheilonema spp., Cercopithifilaria spp., Onchocerca flexuosa, and Mansonella (Esslingeria) perstans. Grey areas are for species or genera studied since Casiraghi et al., 2004; half grey are for species included in previously studied genus (example Onchocerca). Subgenera of Mansonella: (Cu.) Cutifilaria; (M.) Mansonella; (T.) Tetrapetalonema.

and negatively defines the outgroup (Fig. 1). Thelazia is well chosen because the oesophagus is not divided (e.g. no divisions of the three primordial glandular cells). Parafilaria Yorke \& Maplestone, 1926 and Stephanofilaria Ilhe \& Ilhe-Landenberg, 1933, which also have a short undivided oesophagus in adult and infective larva, are not Filarioidea but Thelazioidea (Bain, 2002). The persistence of such primitive oesophageal anatomy places the three genera close to some Seuratoidea Chabaud, Campana-Rouget \& Brygoo, 1959, a complex superfamily in need of molecular analysis.

Setaria and Filaria, both tissular parasistes like all filarioids, suggest that several stems evolved separately. Setariinae have circulating microfilariae (first stage larva) but their adult cephalic end possesses a salient ring and often pointed papillae; a considerable multiplication of nuclei of the glandular part of the oesophagus occurs during the second larval stage, and the length of the buccal capsule of infective larvae reaches $17 \mu \mathrm{m}$ in some species. Filaria has a "smooth" head, eggs containing first stage-larva and no circulating microfilariae. The definition of Filarioidea should be: tissular, circulating microfilariae and heamatophagous arthropod vector, glandular oesophagus in the infective stage. This applies well only to Onchocercidae. The tendency for several groups of Spirurida to become tissular, such as in dracunculids transmitted by aquatic crustaceans, has long been known and, not surprisingly, is confirmed with the molecular analysis (Nadler et al., 2007).

Dating the origin of the diverse branches of filarioids is not possible with the molecular methods used presently because the mitochondrial $12 \mathrm{~S}$ rDNA and coxI genes are at saturation for deep evolutionary time (see discussion in Casiraghi et al., 2001). At present, the scenario proposed is based on the host-range and geographical distribution. Oswaldofilaria, with two very close species parasitic in crocodiles in South America and in South Africa, is proposed as the first filarioid, 140 mya ago (Bain et al., 1984, in Chabaud \& Bain, 1994). However this hypothesis will need a congruence with future molecular datasets based on slow evolving genes.

\section{CONCLUSION}

The Filarioidea, although zoologically a small group of nematodes, have been intensively studied and 1 are still worthy of further investigation. They cause several tropical diseases and the filariae of non-human animals are potential sources for zoonoses. Filarioids have acquired a unique mode of transmission among nematodes, with haematophagous arthropods acting as intermediate hosts and in which a degree of larval morphogenesis occurs. At present Wolbachia is only known 
in this group of nematodes and these endosymbionts are shared with another large group of Ecdysozoa, the arthropods. The genome of the human filaria Brugia malayi is now known (Ghedin et al., 2007) and demonstrates that new genes appeared and others were lost during the long evolution from free-living ancestors, $e$. g. Caenorhabditis elegans genome. It seems thus unlikely that filarioids will return to a free living life style. Field research on filarial biodiversity undertaken by a worldwide network of collaborators, has given access to new specimens which are informative for the evolution of the filarioids and their wolbachiae endosymbionts. Several species (L. yutajensis/L. sigmodontis; Cercopithifilaria spp.) have now been identified which may serve as models to investigate the "mutualistic" relationships at gene level.

\section{ACKNOWLEDGEMENTS}

W e heartily thank our generous colleagues D. Spratt, Australia, and J. Baker U.K., for the time and thought they have given to revision of the manuscript.

\section{REFERENCES}

Anderson R.C. \& Bain O. Keys to genera of the order Spirurida. Part 3. Diplotriaenoidea, Aproctoidea and Filarioidea, in: CIH keys to the nematode parasites of vertebrates. Anderson R.C., Chabaud A.G. \& Willmott S. (eds), Farnham Royal: Commonwealth Agricultural Bureaux, 1976, No. 3, 59-116.

BAIN O. Evolutionary relationships among filarial nematodes, in: World Class Parasites. Klei \& Rajan (eds), Kluwer Acad. Publ., vol. 5, The Filaria 2002, 21-29.

Bain O. \& Chabaud A.G. Atlas des larves infectantes de Filaires. Tropical Medecine and Parasitology, 1986, 37, 237340.

Bain O., Wanji S., Enyong P., Petit G., Noireau F., Eberhard M.I. \& WAHL G. New features on the moults and morphogenesis of the human filaria Loa loa using rodent hosts. Consequences. Parasite, 1998, 5, 37-46.

Bandi C., Anderson T.J.C., Genchi C. \& Blaxter M.L. Phylogeny of Wolbachia in filarial nematodes. Proceeding of the Royal Society, London, Biological Science, 1998, 265, 24072413.

Casiraghi M., Anderson T.J.C., Bandi C., Bazzocchi C. \& GenCHI C. A phylogenetic analysis of filarial nematodes: comparison with the phylogeny of Wolbachia endosymbionts. Parasitology, 2001, 122, 93-103.

Casiraghi M., Bain O., Guerrero R., Martin C., Pocacqua V, Gardner S.L., Franceschi A. \& Bandi C. Mapping the presence of Wolbachia pipientis on the phylogeny of filarial nematodes: evidence for symbiont loss during evolution. International Journal of Parasitology, 2004, 34, 191-203.

Casiraghi M., Bordenstein S.R., Baldo L., Lo N., Beninati T.,
WernegreEn J.J., Werren J.H. \& BANDi C. Phylogeny of Wolbachia pipientis based on gltA, groEL and ftsZ gene sequences: clustering of arthropod and nematode symbionts in the F supergroup, and evidence for further diversity in the Wolbachia tree. Microbiology, 2005, 151, 4015-4022.

Chabaud A.G. \& Bain O. The evolutionary expansion of the Spirurida. International Journal of Parasitology, 1994, 24, 1179-1201.

Eberhard M.L. \& Orihel T.C. The genus Mansonella (Syn. Tetrapetalonema). A new classification. Annales de Parasitologie Humaine et Comparée, 1984, 59, 483-496.

Fenn K., Conlon C., Jones M., Ouall M.A., Parkhill J. \& BlaXTER M. Phylogenetic relationships of the Wolbachia of nematodes and arthropods. PLoS Pathogens, 2006, 2, e94.

Fischer P., Schmetz C., Bandi C., Bonow I., Mand S., Fischer K. \& ButTnER D.W. Tunga penetrans: molecular identification of Wolbachia endobacteria and their recognition by antibodies against proteins of endobacteria from filarial parasites. Experimental Parasitology, 2002, 102, 201-211.

Ghedin E., WAng S., Spiro D. et al. Draft genome of the filarial nematode parasite Brugia malayi. Science, 2007, 21, 317, 1756-1760.

Gorham C.H., FAnG Q.Q. \& DuRden L.A. Wolbachia endosymbionts in fleas (Siphonaptera). Journal of Parasitology, 2003, 89, 283-289.

Grobusch M.P., Kombila M., Autenrieth I., Mehlhorn H. \& Kremsner P.G. No evidence of Wolbachia endosymbiosis with Loa loa and Mansonella perstans. Parasitology Research, 2003, 90, 405-408.

Kruger A., Fischer P. \& Mrales-Hojas R. Molecular phylogeny of the filaria genus Onchocerca with special emphasis on Afrotropical human and bovine parasites. Acta Tropica, 2007, 101, 1-14.

lo N., Casiraghi M., Salati E., Bazzocchi C., Bandi C. How many Wolbachia supergroups exist? Molecular Biology and Evolution, 2002, 19, 341-346.

Nadler S. A., Carreno R.A., Mejia-Madrid H., Ullberg J., Pagan C., Houston R. \& Hugot J.P. Molecular phylogeny of clade III nematodes reveals multiple origins of tissue parasitism. Parasitology, 2007, 134, 1421-1442.

SRÉTER-LANCZ Z., SZÉll Z. \& SRÉTER T. Molecular genetic comparison of Onchocerca sp. infecting dogs in Europe with other spirurid nematodes including Onchocerca lienalis. Veterinary Parasitology, 2007, 148, 365-370.

Uni S., Bain O., Agatsuma T., Katsumi A., Baba M., Yanai T. \& TAKAOKA H. New filarial nematode from Japanese serows (Naemorhedus crispus: Bovidae) close to parasites from elephants. Parasite, 2006, 13, 193-200.

Uni S., Bain O, Agatsuma T., Harada M., Tori H., Fukuda M. \& TAKAOKA H. Onchocerca eberhardi n. sp. (Nematoda: Filarioidea) from the sika deer in Japan; relationhips between species parasitic in cervids and bovids in holarctic region. Parasite, 2007, 14, 199-211.

WEHR E.E. A revised classification of the nematode superfamily Filarioidea. Proceedings of the Helminthological Society of Washington, 1935, 2, 84-88.

Xie H., Bain O. \& Williams S.A. Molecular phylogenetic studies on filarial parasites based on $5 \mathrm{~S}$ ribosomal spacer sequences. Parasite, 1994, 1, 141-151. 